\section{Leptotrichia buccalis: a novel cause of chorioamnionitis}

\author{
Marcela C. Smid,' Sarah K. Dotters-Katz, ${ }^{1}$ \\ Rongpong Plongla, 2,3 Kim A. Boggess ${ }^{1}$ \\ 1 Department of Obstetrics and \\ Gynecology, Division of Maternal Fetal \\ Medicine, ${ }^{2}$ Department of Pathology and \\ Laboratory Medicine, University of North \\ Carolina, Chapel Hill, NC, USA; \\ ${ }^{3}$ Department of Medicine, Chulalongkorn \\ University and King Chulalongkorn \\ Memorial Hospital, Thai Red Cross \\ Society, Bangkok, Thailand
}

\section{Abstract}

Intra-amniotic Leptotrichia buccalis has not been previously associated with adverse pregnancy outcome. We report a case of chorioamnionitis and pregnancy loss associated with this primarily oral commensal. We review Leptotrichia buccalis and other oral commensals that have been identified in cases of intraamniotic infection.

\section{Case Report}

A 35-year-old multigravida woman with a history of loop electrosurgery excision procedure (LEEP) six months prior to index pregnancy, presented at 19 weeks and 1 day for an anatomy ultrasound. At that time, her cervical length was noted to be $9 \mathrm{~mm}$. She was counseled on management options including vaginal progesterone, cerclage, a purse-string stitch placed in the cervix, and expectant management. She elected for expectant management. One week later on a follow-up ultrasound, she had no measurable cervical length and prolapsing membranes through the external os. A sterile speculum examination confirmed the location of the membranes, with no evidence of rupture.

She was afebrile and without fundal tenderness. She had a white blood cell count of $9.9 \times 10^{9} /$ L. Options, including expectant management and cerclage, were discussed with the patient and she desired cerclage placement attempt. Amniocentesis was performed to assess the presence of a sub-clinical infection prior to a cerclage placement. The Gram stain was negative for organisms, the white blood cell count was 9.5 cells $/ \mathrm{mm}^{3}$, and the glucose was $<20 \mathrm{mg} / \mathrm{dL}$, which is the lowest reportable value to which our hospital instruments are validated. Urinalysis showed no sign of infection. A nucleic acid amplification testing for
Chlamydia trachomatis and Neisseria gonorrhoeae (Gen-Probe APTIMA Combo 2 Assay, Hologic, Bedford, MA, USA) was negative. Based on the results of the Gram stain and amniotic fluid WBC as well as the absence of clinical signs of chorioamnionitis, we proceeded with an exam-indicated cerclage the following morning, after administration of indomethacin and cefazolin $1 \mathrm{~g}$ intravenously every eight hours overnight per our institution's protocol. She was discharged home that same day with a plan for close follow up. Amniotic fluid was cultured on chocolate agar, Columbia CNA agar with 5\% sheep blood, MacConkey agar and enriched thioglycollate medium with vitamin $\mathrm{K}$ and hemin (Beckton Dickinson, Franklin Lakes, NJ, USA) at $35^{\circ} \mathrm{C}$ with $5-10 \% \mathrm{CO}_{2}$. Anaerobic culture was also performed on CDC anaerobe 5\% sheep blood agar and anaerobic reducible CNA blood agar (Remel, Lenexa, KS, USA) at $35^{\circ} \mathrm{C}$. We incubated all aerobic media in air with 5-10\% C02 environment (average of 7\%) and this culture grew very well as some strains of Leptotrichia buccalis is aerotolerant and can grow in 5\% C02. ${ }^{1}$

The day following her cerclage placement, the preliminary culture from her amniocentesis returned 1+ filamentous Gram-negative rods, growing only on the chocolate agar plate (Figure 1). The organism was identified as Leptotrichia buccalis with confidence value of $99.7 \%$ using matrix-assisted laser desorption ionization time-of-flight mass spectrometry (MALDI-TOF MS; bioMérieux Vitek MS, Durham, NC, USA) using the v2.0 Knowledge Base database. Then, $16 \mathrm{~S}$ rRNA gene sequencing was performed to confirm the identification as $L$. buccalis, which matched $98.5 \%$ identity by the method described previously. ${ }^{2}$ The patient was called, and she complained of back pain. She was evaluated and had no clinical signs or symptoms consistent with chorioamnionitis. Given her clinically stable picture, she was discharged with precautions and a plan for follow up in four days. Two days later, the patient developed abdominal pain and bleeding. She presented to her local hospital in labor, the cerclage was removed, and she delivered a non-viable infant. She was treated with antibiotics for twenty-four hours and recovered without complication. Placenta pathology was consistent with acute chorioamnionitis.

\section{Discussion}

There is increasing evidence that the gravid uterus is not a sterile environment. Gibbs et al. identified organisms in the amniotic fluid of $31 \%$ of term patients without any evidence of clinical infection. ${ }^{3}$ More recently, Bearfield et al. reported that 34 (71\%) of 48 women deliv-
Correspondence: Marcela Smid, Department of Obstetrics and Gynecology, Division of Maternal Fetal Medicine, University of North Carolina Chapel Hill 3010 Old Clinic Building, CB 7516, Chapel Hill, NC 27599-7516, USA.

Tel.: + 1.919.966.4103 - Fax: +1.919.966.6377.

E-mail: marcela_smid@med.unc.edu

Key words: Leptotrichia; pregnancy complication; chorioamnionitis; microbiome.

Contributions: the authors contributed equally.

Conflict of interest: the authors declare no potential conflict of interest.

Received for publication: 7 January 2015.

Revision received: 27 March 2015.

Accepted for publication: 8 April 2015.

This work is licensed under a Creative Commons Attribution NonCommercial 3.0 License (CC BYNC 3.0).

(C) Copyright M.C. Smid et al., 2015

Licensee PAGEPress, Italy

Infectious Disease Reports 2015; 7:5801

doi:10.4081/idr.2015.5801

ering at term via cesarean section had evidence of bacterial contamination in amniotic fluid through DNA testing, while 21 (44\%) had bacteria present on microscopy. These women had no clinical signs of infection at time of delivery, although 21 (44\%) of 48 reported infections in the index pregnancy including urinary tract infection, bacterial vaginosis, periodontal disease and respiratory infection. ${ }^{4}$ The debate over the significance of identifying bacterial DNA in the amniotic fluid of asymptomatic pregnant women continues. The challenge for the clinicians arises in determining when the presence of bacteria, particularly oral commensals such as L. buccalis, is benign and when it poses a risk to the mother, the fetus, and the pregnancy.

Leptotrichia species are slow-growing, anaerobic gram negative bacteria that are found as part of the endogenous oral, intestinal and genitourinary tract. ${ }^{5,6}$ Only recently have Leptotrichia species been appreciated as cause of human disease. Eribe $e t$ al. have described a myriad of pathological conditions associated with Leptotrichia, including appendicitis, pneumonia, mucositis and sepsis. ${ }^{5}$ L. buccalis, is primarily an oral commensal and has been associated with infections in immunocompromized individuals. ${ }^{7,8}$ Both Leptotrichia amnionii and $L$. sanguinegens have been associated with adverse pregnancy outcomes including miscarriage, chorioamnionitis, preterm labor, neonatal infection and postpartum infection..$^{9-15}$ This case, to our knowledge, is the first reported case of $L$. buccalis associ- 
ated with chorioamnionitis and pregnancy loss. We hypothesize that the LEEP procedure six months prior to pregnancy may have increased risk for cervical incompetence. ${ }^{16}$ Exposed amniotic membranes may have increased the risk of ascending infection from vaginal colonization leading to chorioamnionitis, however, L. buccalis, unlike other Leptrotrichia species, is not typically found in the vagina. An alternative explanation for the development of chorioamnionitis is hematogenous spread from the oral cavity.

A wide variety of organisms, both pathologic and benign, have been identified in the intraamniotic environment, including oral flora. Eikenella corrodens have been described as causative agents in intra-amniotic infection, though these are also known to exist as part of the vaginal flora as well. ${ }^{17}$ Capnocytophaga sputigena, Capnocytophaga leadbetteri, and Fusobacterium nucleatum, are all members of the oral microbiome that are not typically found as part of the vaginal flora, and have been isolated in cases of chorioamnionitis. ${ }^{4,18}$ Bacterial infection causing adverse pregnancy outcomes may be related to cytokine-mediated inflammatory reaction. Bacteria incite inflammation of decidua and/or chorioamnion leading to activation of macrophages. Activated macrophages then release cytokines, most notably IL-6, which initiate prostaglandin secretion, initiating cervical dilation and eventual delivery..$^{19}$ However, the exact pathway by which normal commensals including oral flora, is associated with clinical infections and adverse pregnancy outcomes has yet to be elucidated. When clinicians incidentally encounter evidence of bacterial intra-amniotic infection in the setting of normal pregnancy, clinical judgment and patient preferences should be used to balance the risks and benefits of continuing pregnancy with maternal and fetal well-being. There is some evidence that antibiotic treatment may improve pregnancy outcomes for asymptomatic women even with highly pathogenic bacteria, including Ureaplasma urealyticum or Mycoplasma hominis. ${ }^{20}$ In our case, prior to cerclage placement, the patient did receive cefazolin, a first generation cephalosporin, which have been shown to be successful in the treatment of Leptotrichia in non-obstetrical infection. ${ }^{8,21,22}$

\section{Conclusions}

In summary, clinicians encountering pregnancies complicated by the presence of intrauterine commensals, not previously associated with intra-amniotic infection, should report their findings and pregnancy outcome. Disseminating this information may help guide management and counseling for other

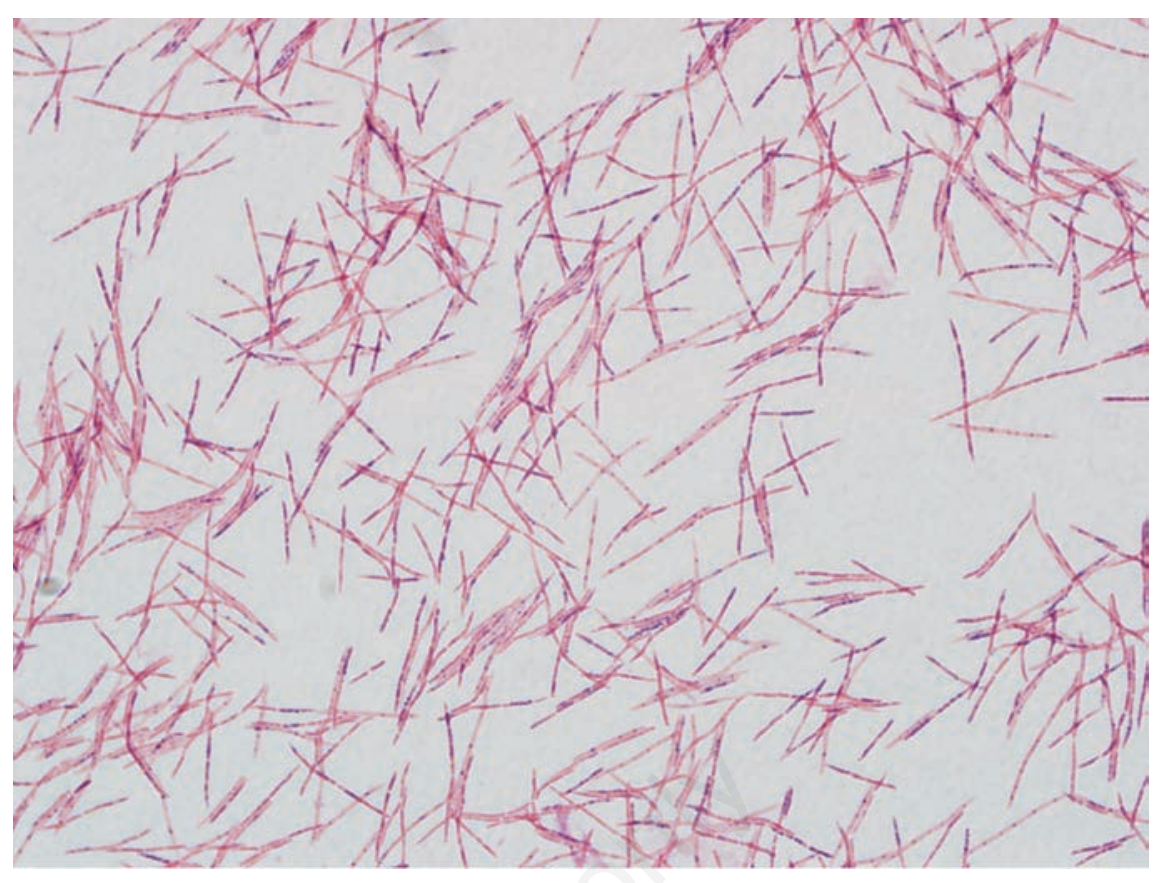

Figure 1. Leptotrichia buccalis.

patients in the future. With regard to counseling patients on follow-up of asymptomatic cervical dilation treated with exam-indicated cerclage, clinicians should consider any evidence of intra-amniotic bacterial contamination, including oral flora such as Leptotrichia buccalis, an ominous sign.

\section{References}

1. Bernard K, Cooper C, Tessier S, Ewan EP. Use of chemotaxonomy as an aid to differentiate among Capnocytophaga species, CDC group DF-3, and aerotolerant strains of Leptotrichia buccalis. J Clin Microbiol 1991;29:2263-5.

2. Woo PC, Ng KH, Lau SK, et al. Usefulness of the MicroSeq $50016 \mathrm{~S}$ ribosomal DNAbased bacterial identification system for identification of clinically significant bacterial isolates with ambiguous biochemical profiles. J Clin Microbiol 2003;41:19962001.

3. Gibbs RS, Blanco JD, St Clair PJ, Castaneda YS. Quantitative bacteriology of amniotic fluid from women with clinical intraamniotic infection at term. J Infect Dis 1982;145:1-8.

4. Bearfield C, Davenport ES, Sivapathasundaram V, Allaker RP. Possible association between amniotic fluid microorganism infection and microflora in the mouth. BJOG 2002;109:527-33.
5. Eribe ER, Olsen I. Leptotrichia species in human infections. Anaerobe 2008;14:1317.

6. Zhou X, Bent SJ, Schneider MG, et al. Characterization of vaginal microbial communities in adult healthy women using cultivation-independent methods. Microbiology (Reading, England) 2004;150:2565-73.

7. Lark RL, McNeil SA, VanderHyde K, et al. Risk factors for anaerobic bloodstream infections in bone marrow transplant recipients. Clin Infect Dis 2001;33:338-43.

8. Weinberger M, Wu T, Rubin M, et al. Leptotrichia buccalis bacteremia in patients with cancer: report of four cases and review. Rev Infect Dis 1991;13:201-6.

9. Shukla SK, Meier PR, Mitchell PD, et al. Leptotrichia amnionii sp. nov., a novel bacterium isolated from the amniotic fluid of a woman after intrauterine fetal demise. $\mathrm{J}$ Clin Microbiol 2002;40:3346-9.

10. De Martino SJ, Mahoudeau I, Brettes JP, et al. Peripartum bacteremias due to Leptotrichia amnionii and Sneathia sanguinegens, rare causes of fever during and after delivery. J Clin Microbiol 2004;42: 5940-3.

11. Boennelycke M, Christensen JJ, Arpi M, Krause S. Leptotrichia amnionii found in septic abortion in Denmark. Scand J Infect Dis 2007;39:382-3.

12. Thilesen CM, Nicolaidis M, Lokebo JE, et al. Leptotrichia amnionii, an emerging pathogen of the female urogenital tract. $\mathbf{J}$ 
Clin Microbiol 2007;45:2344-7.

13. Decroix V, Goudjil S, Kongolo G, Mammeri H. Leptotrichia amnionii, a newly reported cause of early onset neonatal meningitis. J Med Microbiol 2013;62:785-8.

14. Hanff PA, Rosol-Donoghue JA, Spiegel CA, et al. Leptotrichia sanguinegens sp. nov., a new agent of postpartum and neonatal bacteremia. Clin Infect Dis 1995;20:S2379.

15. Gardella C, Riley DE, Hitti J, et al. Identification and sequencing of bacterial rDNAs in culture-negative amniotic fluid from women in premature labor. Am J Perinatol 2004;21:319-23.

16. Conner SN, Cahill AG, Tuuli MG, et al.
Interval from loop electrosurgical excision procedure to pregnancy and pregnancy outcomes. Obstet Gynecol 2013;122:11549.

17. Andres MT, Martin MC, Fierro JF, Mendez FJ. Chorioamnionitis and neonatal septicaemia caused by Eikenella corrodens. J Infect 2002;44:133-4.

18. McDonald H, Gordon DL. Capnocytophaga species: a cause of amniotic fluid infection and preterm labour. Pathology 1988;20:746.

19. Andrews WW, Goldenberg RL, Hauth JC. Preterm labor: emerging role of genital tract infections. Infect Agents Dis 1995;4:196-211.
20. Berg TG, Philpot KL, Welsh MS, et al. Ureaplasma/mycoplasma-infected amniotic fluid: pregnancy outcome in treated and nontreated patients. J Perinatol 1999;19: 275-7.

21. Gundi VA, Desbriere R, La Scola B. Leptotrichia amnionii and the female reproductive tract. Emerg Infect Dis 2004;10:2056-7.

22. Morgenstein AA, Citron DM, Orisek B, Finegold SM. Serious infection with Leptotrichia buccalis. Report of a case and review of the literature. Am J Med 1980; 69:782-5. 